\title{
Physical Function in Subjects Requiring Extracorporeal Membrane Oxygenation Before or After Lung Transplantation
}

\author{
Kate Hayes PT MPhysio, Carol L Hodgson PT PhD, Vincent A Pellegrino MD, Greg Snell MD, \\ Benjamin Tarrant PT, Louise M Fuller PT, and Anne E Holland PT PhD
}

\begin{abstract}
BACKGROUND: Extracorporeal membrane oxygenation (ECMO) is used as a rescue therapy before and after lung transplantation, but little is known about functional recovery or complications after ECMO in this cohort. This study aimed to describe early physical function and leg complications in subjects who received ECMO before or after lung transplantation, and to compare functional outcomes to a matched cohort of subjects who did not require ECMO. METHODS: A retrospective study was conducted over 2 years. Highest mobility level was assessed, in both the ECMO and non-ECMO groups, prior to ICU admission, at ICU discharge, and at hospital discharge, while 6-min walk distance was measured at hospital discharge and at 3 months. Strength was assessed at ICU discharge and at hospital discharge in the ECMO subjects only, and leg complications were recorded up until hospital discharge. RESULTS: 17 subjects (mean age $43 \pm 13$ y; 65\% (11 of 17 subjects) female) required ECMO before or after lung transplant. Survival to hospital discharge was $82 \%$ (14 of 17 subjects). At ICU discharge, strength and mobility levels were poor, but both improved by hospital discharge $(P<.001)$. Leg complications were reported in $50 \%$ of survivors (7 of 14 subjects). ECMO survivors spent longer in the ICU $(P<.001)$ and hospital $(P=.002)$ and had worse physical function (ie, lower mobility level at ICU discharge, mean difference $-1, P=.02 ; 6$-min walk distance at hospital discharge: mean difference $-99 \mathrm{~m}, P=.004$ ) than lung transplant recipients not requiring ECMO $(n=28)$. CONCLUSIONS: In subjects requiring ECMO before or after lung transplantation, $82 \%$ survived to hospital discharge, but leg complications were common and physical function was poor at ICU discharge. Physical function improved over time, however subjects who required ECMO had a longer period of hospitalization and worse physical function at ICU and hospital discharge than those who did not require ECMO. Key words: extracorporeal membrane oxygenation; lung transplantation; rehabilitation; quality of life. [Respir Care 2018;63(2):194-202. (C) 2018 Daedalus Enterprises]
\end{abstract}

\section{Introduction}

Lung transplantation is an established therapy for select patients with end-stage lung disease. The number of lung

\footnotetext{
Drs Hodgson and Holland, as well as Ms Hayes, Mr Tarrant, and Ms Fuller are affiliated with the Department of Physiotherapy, The Alfred Hospital, Melbourne, Victoria, Australia. Dr Holland, Ms Hayes, Mr Tarrant, and Ms Fuller are affiliated with the Discipline of Physiotherapy, La Trobe University, Melbourne, Victoria, Australia. Dr Hodgson is affiliated with the Australian and New Zealand Intensive Care Research Centre, Department of Epidemiology and Preventive Medicine, Monash University, Melbourne, Australia. Dr Pellegrino is affiliated with the Intensive Care Department, The Alfred Hospital, Melbourne, Victoria, Australia. Dr Snell is affiliated with the Lung Transplant Service, The Alfred Hospital, Melbourne, Victoria, Australia.
}

transplants performed worldwide has steadily increased, ${ }^{1}$ with the demand for lung transplantation outnumbering the donor organ supply, resulting in waiting list mortality.

\footnotetext{
Ms Hayes presented a version of this paper at the 36th Annual Meeting of the International Society for Heart and Lung Transplantation, on April 29, 2016, in Washington, DC.

The authors have disclosed no conflicts of interest.

Correspondence: Kate Hayes PT MPhysio, Physiotherapy Department, The Alfred Hospital, 55 Commercial Road, Melbourne, Victoria, Australia 3181. E-mail: k.hayes@alfred.org.au.
}

DOI: $10.4187 /$ respcare. 05334 


\section{Physical Function Post ECMO}

In this setting, extracorporeal membrane oxygenation (ECMO) may be considered as a bridge to lung transplantation until compatible donor lungs become available.

ECMO provides temporary support of heart and/or lung function by a modified cardiopulmonary bypass machine. ${ }^{2}$ It can be inserted in a venovenous configuration for pure respiratory support or in a venoarterial configuration for combined cardiac and respiratory support. Early reports of ECMO as a bridge to lung transplantation were not favorable, ${ }^{3}$ and many centers considered ECMO and mechanical ventilation as a contraindication to lung transplantation. In recent years, with significant improvement in ECMO technology, several studies have shown promising outcomes related to the use of ECMO as a bridging strategy $^{4-6}$ as well as a rescue therapy after lung transplantation for primary graft dysfunction or other complications such as severe pulmonary hypertension. ${ }^{7,8}$

ECMO may involve cannulation of the femoral vessels, and lower limb sequelae have been reported in patients who required ECMO before or after heart transplantation. ${ }^{9}$ These leg complications may have an impact on physical function, participation in rehabilitation, and health-related quality of life (HRQOL), but to date they have not been reported in patients before or after lung transplantation with differing ECMO configurations. Although survival after ECMO has improved, little is known about the early physical function of patients who require ECMO before or after lung transplantation, and whether it differs from transplant recipients not requiring ECMO. In addition, there may be a difference between the physical function outcomes of patients who require ECMO before versus after lung transplantation. This information may assist in the development of targeted treatment strategies and modification of potential risk factors for future complications.

The aims of this study were to describe early physical function and leg complications in subjects who received ECMO before or after lung transplantation, and to compare physical function with lung transplant recipients not requiring ECMO.

\section{Methods}

A retrospective, single-center study was conducted between September 2012 and September 2014 at the Alfred Hospital in Melbourne, Australia, a tertiary referral hospital for ECMO in Australia, which also provides a lung transplantation program. This work was supported by an Australian Government Research Training Program Scholarship. This study received local ethics approval. Data were extracted from the prospectively updated physiotherapy ECMO database and hospital transplant database. Consecutive subjects, aged $>18$ y who received ECMO before or after lung transplantation were included. Patients were excluded if they did not meet the criteria for ECMO

\section{QUICK LOOK}

\section{Current knowledge}

Extracorporeal membrane oxygenation (ECMO) is increasingly being used as a rescue therapy for patients both prior to and after lung transplantation. Survival after ECMO in this population has improved, but little is known about the early physical function, leg complications, or health-related quality of life of survivors and whether it differs between those requiring ECMO before versus after transplant. It is also unclear whether physical function outcomes are different for lung transplant patients not requiring ECMO.

\section{What this paper contributes to our knowledge}

Subjects requiring ECMO before or after lung transplantation had very poor muscle strength and mobility levels at ICU discharge. Physical function improved by hospital discharge and continued to improve after discharge from hospital. Lung transplant recipients requiring ECMO required longer periods of mechanical ventilation, spent longer in the ICU and hospital, and had worse physical function than non-ECMO subjects. ICU stay was the only significant predictor of physical function at hospital discharge. Vascular and sensory neurological leg complications were common in subjects who underwent ECMO via femoral vessel cannulation and accounted for $10 \%$ of hospital readmissions in the first year after lung transplantation.

and lung transplantation, including the presence of any additional severe chronic organ failure (liver, cardiac, renal), acute brain injury, recent malignancy, age $>70 \mathrm{y}$, any other contraindication to lung transplantation or reversible respiratory failure not necessitating listing for lung transplantation. All lung transplant patients who did not receive ECMO over the same time period were identified from the hospital transplant database and formed a comparison group for functional outcomes. They were matched (2:1) with the ECMO group for age $( \pm 5 \mathrm{y})$ and gender.

\section{ECMO Criteria and Configuration}

The decision to use ECMO was made by a team composed of ECMO-trained intensive care specialists, lung transplant physicians, and cardiothoracic surgeons. Criteria for the use of ECMO were according to established hospital protocols. ${ }^{10}$ Choice of ECMO configuration was determined by the clinical need and anatomical limitations of each subject. In hemodynamically stable subjects, venovenous ECMO was provided via a dual-lumen cannula 


\section{Physical Function Post ECMO}

(Avalon, Maquet-Getinge, Rastatt, Germany) in the right internal jugular vein ${ }^{11}$ or percutaneously placed femorofemoral cannulae (Medtronic, Minneapolis, Minnesota) inserted under ultrasound guidance. Where concomitant cardiac support was required, peripheral venoarterial ECMO was delivered through percutaneously placed femoro-femoral cannulae under ultrasound guidance. This routinely included insertion of an ante-grade 8.5 French distal perfusion cannula (Mayo, Rochester, Minnesota) at the time of ECMO commencement to prevent limb ischemia. Femoral artery cannulation sites were repaired surgically after decannulation. The ECMO circuit consisted of a Jostra Rotaflow centrifugal pump (Maquet-Getinge) and a Quadrox oxygenator (Maquet-Getinge).

\section{Routine Care and Medications}

Our approach to steroid use before lung transplant is to prescribe steroids only for patients with pulmonary fibrosis or those requiring re-transplant; and these prescriptions are low in dose $(<10 \mathrm{mg}$ prednisolone per day). After lung transplant, all subjects received steroids $(2 \times 500 \mathrm{mg}$ prednisolone intra-operatively, $150 \mathrm{mg}$ on day 1 postoperatively and $1 \mathrm{mg} / \mathrm{kg}$ thereafter, reducing to $20 \mathrm{mg}$ by $5 \mathrm{mg}$ per day, and typically still at $15 \mathrm{mg}$ by 3 months).

At our center, ECMO prior to lung transplantation requires that patients be awake and spontaneously breathing without ventilator support, therefore sedation and neuromuscular blockers were not used routinely. Subjects who required ECMO after transplantation were universally sedated and mechanically ventilated to facilitate safe lung ventilation. Daily sedation targets were generally aimed at a Richmond Agitation and Sedation Scale (RASS) ${ }^{12}$ score of 0 to -2 with daily sedation breaks. Continuous neuromuscular blockers were not routine, although short-term paralysis was occasionally used to facilitate procedures where required. A retrospective review of the medical history was used to check for adherence to the above protocol.

\section{Clinical and Demographic Characteristics}

Demographic and clinical characteristics were recorded from the database and included age, gender, etiology of lung disease, ECMO type and duration, ICU and hospital length of stay (LOS), and in-hospital mortality. The Acute Physiology and Chronic Health Evaluation II (APACHE II) score was calculated at ICU admission. Development of organ system dysfunction was recorded during the ICU stay from a retrospective review of the medical histories. Multiorgan dysfunction syndrome was defined as the concurrent dysfunction of 2 or more organ systems not involved in the disorder that resulted in ICU admission. ${ }^{13}$ Hospital readmissions, LOS, and cause for readmission were recorded for the first year after lung transplantation.
Scheduled readmissions for routine follow-up (eg, endobronchial biopsy) were excluded.

\section{Physical Function}

All physical function data were collected according to standardized protocols by trained physiotherapists. The highest level of subject mobility was recorded in the week prior to ICU admission, and at ICU and hospital discharge using the ICU mobility scale (IMS). This was recorded in both the ECMO and non-ECMO lung transplant groups. The IMS measures the highest level of mobility on a scale of 0 to 10 , with 10 being the best score. ${ }^{14}$ While on ECMO, the highest level of mobility achieved was also recorded using the IMS. Where subjects continued on to transplant, 6-min walk distance (6MWD) was measured using a standardized procedure ${ }^{15}$ at hospital discharge and at 3 months after discharge, and results were compared between the ECMO and non-ECMO lung transplant groups. Muscle strength was assessed in the ECMO subjects using the Medical Research Council sum-score (MRC) at ICU and hospital discharge. The MRC includes isometric strength assessment of 3 upper limb and 3 lower limb muscle groups bilaterally on a $0-5$-point ordinal scale to obtain a maximum score of $60 .{ }^{16} \mathrm{~A}$ score of $<48$ indicates ICU-acquired weakness. ${ }^{17,18}$ Muscle strength was not assessed in the non-ECMO cohort.

Standard care rehabilitation before lung transplant consisted of out-patient supervised exercise training classes 2-3 times a week while on the transplant waiting list. The content of these sessions was based on established pulmonary rehabilitation guidelines. ${ }^{19}$

Our standard rehabilitation program for patients after lung transplant, including those requiring ECMO, was initiated in the ICU as early as the first postoperative day with the goal of achieving the highest level of mobility each day and progressing to ambulation where possible. While on ECMO, rehabilitation began with resistance and range of motion exercises for the upper and lower limbs, progressing to sitting, standing, and, ultimately, ambulation, as medical stability allowed. When patients were discharged from ICU after lung transplantation and were able to independently mobilize on the ward, they commenced 12 weeks of supervised, gym-based, aerobic and strengthening exercises for $1 \mathrm{~h}, 3$ times a week.

\section{Leg Complications}

Leg complications were recorded from the time of ECMO commencement until hospital discharge and included vascular complications (eg, multiple vascular repairs, fasciotomy, embolectomy, seroma requiring repeated drainage or surgical intervention and limb amputation during or after ECMO) and neurologic complications (eg, defined as 
motor or sensory deficit on neurological exam, abnormal nerve conduction study, magnetic resonance imaging).

\section{Health-Related Quality of Life}

HRQOL was assessed in the ECMO group at hospital discharge using the Short-Form General Health Survey (SF-36) Version 2. The SF-36 yields 8 domain scores: physical functioning, role physical, bodily pain, general health, vitality, social functioning, role emotional, and mental health. The 8 domain scores are combined into 2 norm-based summary measures, providing overall estimates of physical health (physical component score) and mental health (mental component score). Domain scores are presented as percentage scores ranging from 0 (worst) to 100 (best), and norm-based scores are standardized for population data, where mean \pm SD is $50 \pm 10 .{ }^{20}$ To illustrate the degree of impairment and the domains particularly affected, median SF-36 scores were compared with Australian normative values. ${ }^{21}$ HRQOL was not assessed in the non-ECMO group.

\section{Statistical Analysis}

Statistical analysis was performed using IBM SPSS Statistics Version 22 for Windows (SPSS, Chicago, Illinois). Continuous variables were expressed as mean $\pm \mathrm{SD}$, and ordinal variables were presented as medians [interquartile ranges (IQR)]. Comparison between groups was performed using independent $t$ tests for continuous data or MannWhitney $\mathrm{U}$ tests for nonparametric continuous variables. The chi-square or Fisher exact test was used for categorical variables. To investigate change over time, a paired $t$ test was performed for continuous data or the Wilcoxon signed-rank test for nonparametric data. Results were considered statistically significant at $P<.05$. Univariate analyses were undertaken to determine the association between each potential predictor variable and 6MWD at hospital discharge. Variables demonstrating $P<.2$ on univariate analysis were entered into a multiple linear regression model. When variables were found to be collinear (eg, ICU LOS, ventilation days, hospital LOS), only one was included in the model (ICU LOS).

\section{Results}

Over the 2-year study period, a total of 117 patients underwent ECMO support, of which 17 (15\%) received ECMO either before or after lung transplantation; 7 before lung transplant and 10 after lung transplant (Fig. 1). The majority of subjects ( 15 of 17 , or $88 \%$ ) underwent femoral vessel cannulation (Table 1). The median duration of mechanical ventilation was $7.5 \mathrm{~d}$ (IQR 4.0-15.0). Three subjects ( 1 with ECMO before lung transplant and 2 with ECMO after lung transplant) had multiorgan dysfunction

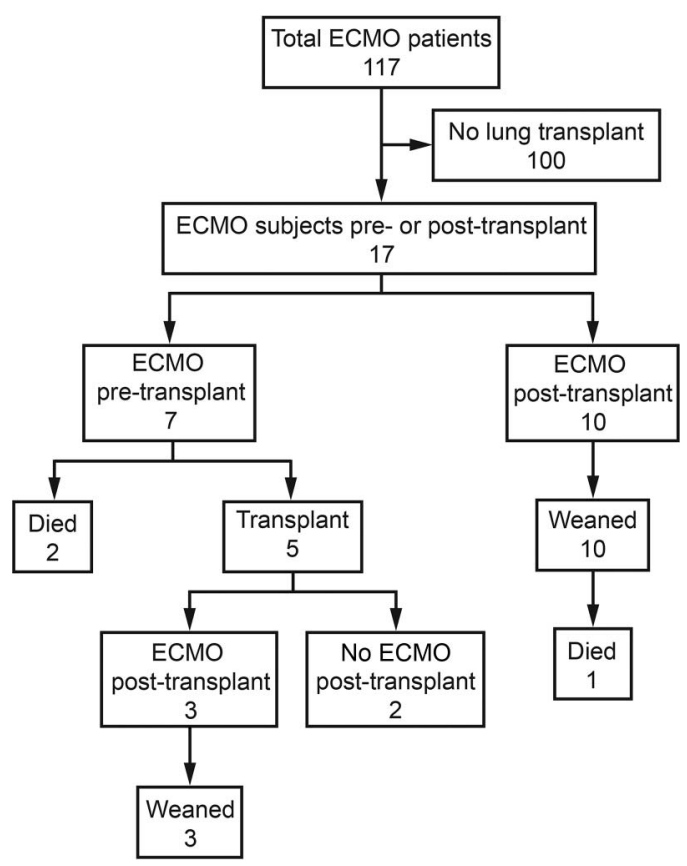

Fig. 1. Flow chart. ECMO = extracorporeal membrane oxygenation.

syndrome. Subjects who underwent ECMO before lung transplant had a higher APACHE II score than subjects receiving ECMO after lung transplant (Table 1). Of the 7 subjects who received ECMO before lung transplant, 2 required deep sedation (RASS $=-4$ ), intubation, and ventilation and became ineligible for transplant and were palliated. The remaining 5 subjects were managed per the routine sedation and paralysis protocol before lung transplant. Six of the subjects requiring ECMO after lung transplant required deep sedation $($ RASS $=-4$ ) while on ECMO, with the remaining 4 subjects having a RASS score between -1 and -2 as per protocol; none received continuous neuromuscular blockers after transplant.

Overall survival to hospital discharge was $82 \%(n=14)$; 2 subjects died while on ECMO prior to lung transplant, and 1 subject died after lung transplant in the ICU from complications unrelated to ECMO. All subjects who survived to hospital discharge were alive 1 year after lung transplantation.

In the week prior to ICU admission, all subjects who required ECMO after lung transplant $(n=10)$ were ambulating independently with or without a gait aide, and 5 subjects were attending pre-transplant rehabilitation. In comparison, subjects who required ECMO before lung transplant were more debilitated; 1 subject was bed-bound in the $48 \mathrm{~h}$ prior to start of ECMO, 2 subjects were limited to transferring from the bed to an armchair, while the remaining 4 subjects were ambulating with or without a gait aide on the ward. All of the non-ECMO subjects 


\section{Physical Function Post ECMO}

Table 1. Demographic and Clinical Details of Subjects Who Underwent ECMO Before or After Lung Transplantation

\begin{tabular}{|c|c|c|c|}
\hline & ECMO Before Lung Transplant $(n=7)$ & ECMO After Lung Transplant $(n=10)$ & $P$ \\
\hline Age, y & $42.1 \pm 11.5$ & $44.1 \pm 14.2$ & .77 \\
\hline Female, $n(\%)$ & $5(71)$ & $6(60)$ & $>.99$ \\
\hline ECMO duration, $\mathrm{d}$ & $10.0(4.5-16.0)$ & $5.0(4.0-6.0)$ & .35 \\
\hline ICU LOS, d & $17.0(7.5-24.0)$ & $12.0(8.0-36.0)$ & .85 \\
\hline Hospital LOS, d & $48.8 \pm 25.5$ & $35.2 \pm 16.1$ & .24 \\
\hline APACHE II & $24.0(20.0-25.5)$ & $14.5(14.0-17.0)$ & .001 \\
\hline Diagnosis, $n(\%)$ & & & .07 \\
\hline Cystic fibrosis/bronchiectasis & $4(57)$ & $1(10)$ & \\
\hline COPD, asthma, and obliterative bronchiolitis & $1(14)$ & $4(40)$ & \\
\hline Pulmonary hypertension & $0(0)$ & $4(40)$ & \\
\hline Pulmonary fibrosis & $1(14)$ & $1(10)$ & \\
\hline Re-transplant & $1(14)$ & $0(0)$ & \\
\hline ECMO type, $n(\%)$ & & & .02 \\
\hline Venoarterial femoral & $1(14)$ & $8(80)$ & \\
\hline Venovenous femoral & $4(57)$ & $2(20)$ & \\
\hline Venovenous dual lumen internal jugular vein & $2(29)$ & $0(0)$ & \\
\hline In-hospital mortality, $n(\%)$ & $2(29)$ & $1(10)$ & .54 \\
\hline Discharge destination, $n(\%)$ & & & .51 \\
\hline Home & $5(71)$ & $7(70)$ & \\
\hline In-patient rehabilitation & $0(0)$ & $2(20)$ & \\
\hline
\end{tabular}

Values are presented as mean $\pm \mathrm{SD}$, median (interquartile range), or as a number $(\%) . P$ values represent the difference between before- and after-transplant groups.

$\mathrm{ECMO}=$ extracorporeal membrane oxygenation

LOS = length of stay

APACHE II = Acute Physiology and Chronic Health Evaluation II

$(n=28)$ were ambulating independently with or without a gait aide in the week prior to ICU admission.

All subjects who required ECMO after lung transplant $(n=10)$ were sedated, ventilated, and resting in bed for the duration of ECMO, and they received only passive range of motion exercises of the upper and lower limbs. Two subjects who required ECMO before lung transplant mobilized out of bed while on ECMO, either to transfer from the bed to a chair or to march in place at the bedside. Both of these subjects had ECMO cannulation of the neck vessels via a dual-lumen cannula. Two more subjects who required ECMO before lung transplant were awake on ECMO and participated in active strength exercises of the upper and lower limbs in bed, while the remaining 3 subjects received passive range of motion exercises as they were sedated or medically unstable for the duration of ECMO. The main reasons for not mobilizing out of bed while on ECMO were venoarterial femoral cannulation, deep sedation, mechanical ventilation, and medical instability.

Lung transplant subjects who required ECMO had more days of mechanical ventilation and a longer ICU and hospital LOS than those who did not require ECMO (Table 2). The highest mobility level achieved at ICU discharge was also lower in the ECMO lung transplant group (IMS median 6) compared to the non-ECMO group (IMS median 7). This translates to the ECMO lung transplant subjects being limited to marching in place at the bedside versus the non-ECMO subjects ambulating away from the bedside with the assistance of 2 or more people at ICU discharge. This improved by hospital discharge in both groups (median $=10)$ to a level where subjects were independently walking without a gait aide (Table 2). There was more variability in the mobility levels of subjects in the ECMO group (IMS 7-10) compared to the non-ECMO group (IMS 9-10). The ECMO group took longer to reach mobility milestones than the non-ECMO group.

The ECMO lung transplant cohort had a lower 6MWD at hospital discharge (mean difference $-99 \mathrm{~m}, 95 \% \mathrm{CI}$ -33 to $-165, P=.004)$. In a multiple regression analysis that included ICU LOS, group (ECMO vs no ECMO), and diagnosis (cystic fibrosis vs no cystic fibrosis), a longer ICU LOS was the only significant predictor of lower 6MWD at hospital discharge (standardized beta $=-.50, P=.004$ ). Use of ECMO was not an independent predictor in this model (standardized beta $=0.11, P=.50$ ), reflecting the much longer ICU LOS in the ECMO group (Table 2). Both groups had significantly improved their 6MWD by 3 months after discharge $(P<.001)$, with no differences between groups at this time point.

Muscle strength at ICU discharge was poor in the ECMO cohort (MRC score $44 \pm 10$ ), with $64 \%$ of survivors ( 9 of 14 subjects) having an MRC strength score of $<48 / 60$, indicating ICU-acquired weakness. Muscle strength improved by hospital discharge but remained below normal 


\section{Physical Function Post ECMO}

Table 2. Lung Transplant Survivors Who Underwent EMCO vs No ECMO, Matched for Age and Gender

\begin{tabular}{|c|c|c|c|}
\hline & $\operatorname{ECMO}(n=14)$ & No ECMO $(n=28)$ & $P$ \\
\hline Age, y & $41.8 \pm 12.8$ & $41.2 \pm 13.4$ & .90 \\
\hline Female, $n(\%)$ & $8(57.1)$ & $16(57.1)$ & $>.99$ \\
\hline \multicolumn{4}{|l|}{ Hospital data } \\
\hline APACHE II & $16.5(14.0-20.0)$ & $14.0(12.0-20.0)$ & .16 \\
\hline Ventilation, $\mathrm{d}$ & $5.0(3.5-14.0)$ & $1.0(1.0-1.5)$ & $<.001$ \\
\hline ICU LOS, d & $15.0(8.0-26.0)$ & $5.0(3.0-7.5)$ & $<.001$ \\
\hline Hospital LOS, d & $40.1 \pm 20.1$ & $19.2 \pm 5.8$ & .002 \\
\hline Diagnosis, $n(\%)$ & & & .07 \\
\hline Cystic fibrosis/bronchiectasis & $5(35.7)$ & $18(64.3)$ & \\
\hline COPD, asthma, and obliterative bronchiolitis & $4(28.6)$ & $5(17.9)$ & \\
\hline Pulmonary hypertension & $4(28.6)$ & $1(3.6)$ & \\
\hline Pulmonary fibrosis & $1(7.1)$ & $4(14.3)$ & \\
\hline \multicolumn{4}{|l|}{ Physical function } \\
\hline IMS ICU at discharge & $6(5-7)$ & $7(6-8)$ & .02 \\
\hline IMS at hospital discharge & $10(9-10)$ & $10(10-10)$ & .006 \\
\hline $6 \mathrm{MWD}$ at hospital discharge, $\mathrm{m}$ & $285 \pm 112$ & $384 \pm 93$ & .004 \\
\hline $6 \mathrm{MWD}$ at 3 months, m & $541 \pm 133$ & $584 \pm 67$ & .32 \\
\hline Discharge destination, $n(\%)$ & & & .11 \\
\hline Home & $12(85.7)$ & $28(100)$ & \\
\hline In-patient rehabilitation & $2(14.3)$ & $0(0)$ & \\
\hline
\end{tabular}

Values are presented as mean $\pm \mathrm{SD}$, median (interquartile range), or as a number (\%). $P$ values represent the difference between ECMO versus no ECMO groups $\mathrm{ECMO}=$ extracorporeal membrane oxygenation

APACHE II = Acute Physiology and Chronic Health Evaluation II

LOS $=$ length of stay

IMS $=$ ICU mobility scale

$6 \mathrm{MWD}=6-\mathrm{min}$ walk distance

levels (mean improvement MRC 11, 95\% CI 7-15, $P<.001)$. There was no significant difference in physical function outcomes between subjects who underwent ECMO before versus after lung transplantation.

Leg complications were observed in 50\% of ECMO survivors (7 of 14 subjects), including vascular and sensory neurological injuries (Table 3). The vascular injuries occurred in 6 of 9 subjects who underwent femoral venoarterial ECMO, while the neurological injuries were seen in subjects who underwent femoral venoarterial or venovenous ECMO. The neurological injuries were confined to sensory deficits, with no motor deficits noted. There was no significant difference in 6MWD or SF-36 scores between subjects who had a leg complication compared to those who had no complication. All survivors who had a leg complication were able to complete the 12-week post-transplant rehabilitation program, with only minor modifications required; lower limb resistance exercises including squats and leg press exercises were removed for those with vascular complications.

Subjects undergoing ECMO had lower SF-36 scores at hospital discharge than Australian norms across all domains except role emotional (Fig. 2). The SF-36 physical component score was also lower in our cohort than Australian norms (mean difference $=24.07, P=.003$ ), while there was no difference for the mental component score.
Table 3. Leg Complications After ECMO

\begin{tabular}{|c|c|c|}
\hline Subject & $\begin{array}{l}\text { ECMO } \\
\text { Type }\end{array}$ & Leg Complication \\
\hline 1 & Venoarterial & $\begin{array}{l}\text { Left femoral artery thrombectomy and vein } \\
\text { patch repair of false aneurysm }\end{array}$ \\
\hline 2 & Venoarterial & $\begin{array}{l}\text { Right femoral artery multiple vascular } \\
\text { surgeries, ilio-popliteal bypass, ischemic } \\
\text { right foot with ongoing infection leading } \\
\text { to amputation of toes }\end{array}$ \\
\hline 3 & Venoarterial & $\begin{array}{l}\text { Left groin seroma with long-term drain } \\
\text { in situ; dense paresthesia left thigh }\end{array}$ \\
\hline 4 & Venoarterial & $\begin{array}{l}\text { Right groin hematoma and infection; right } \\
\text { femoral artery reconstruction and vein } \\
\text { patch repair }\end{array}$ \\
\hline 5 & Venoarterial & $\begin{array}{l}\text { Stenosis of external iliac vein resulting in } \\
\text { significant left leg edema, managed } \\
\text { conservatively }\end{array}$ \\
\hline 6 & Venovenous & Right leg paresthesia and neurogenic pain \\
\hline 7 & Venoarterial & $\begin{array}{l}\text { Right groin seroma and right thigh } \\
\text { numbness, bilateral pins and needles }\end{array}$ \\
\hline
\end{tabular}

The majority of ECMO survivors $(79 \%, 11$ of 14 subjects) required hospital readmission within the first year after lung transplantation, with 39 readmissions in total, 


\section{Physical Function Post ECMO}

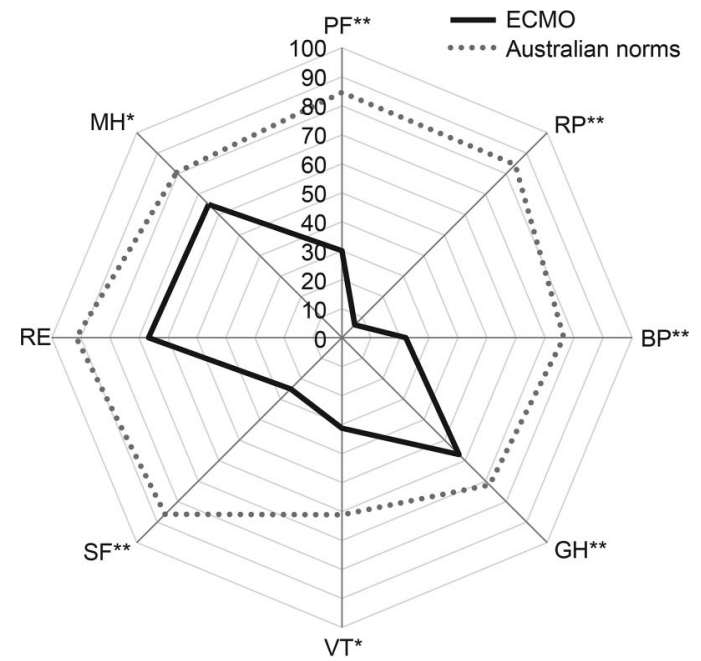

Fig. 2. Comparison of SF-36 scores in lung transplant subjects who received extracorporeal membrane oxygenation $(\mathrm{ECMO}, n=$ 11) versus Australian population norms (data from Reference 20, $N=3,015)$. SF-36, Short-Form General Health Survey (version 2); $\mathrm{PF}$, physical functioning; RP, role physical; $\mathrm{BP}$, bodily pain; $\mathrm{GH}$, general health; VT, vitality; SF, social functioning; RE, role emotional; $\mathrm{MH}$, mental health. ${ }^{\star \star} P<.005,{ }^{\star} P<.05$.

including 8 subjects who required multiple readmissions. The median number of readmissions was 2 (IQR 1-4) with a median LOS of $7 \mathrm{~d}$ (IQR 4-17). The causes for readmission were chest infection (36\%, 14 of 39 subjects), rejection ( $21 \%, 8$ of 39 readmissions), lower limb vascular complications related to ECMO (10\%, 4 of 39 readmissions), and miscellaneous (33\%, 13 of 39 readmissions).

\section{Discussion}

To our knowledge, this is the first study to describe early physical function outcomes in subjects undergoing ECMO before or after lung transplantation, compared to those who did not require ECMO. The majority of the ECMO subjects demonstrated severe muscle weakness at ICU discharge, indicative of ICU-acquired weakness. The cause of this weakness is likely multifactorial, with an interplay between preexisting muscle weakness associated with chronic lung disease ${ }^{22,23}$ and critical illness requiring ECMO. Pre-morbid weakness prior to ECMO may be a factor; however, only 1 subject was bed-bound in the $48 \mathrm{~h}$ prior to ECMO commencement, and the majority of subjects were ambulating independently. The grading of muscle weakness prior to the initiation of ECMO is unknown because this was not objectively assessed due to the acute presentation of subjects to ICU.

A number of factors have been associated with the development of ICU-acquired weakness ${ }^{18}$ and may be related to the worse physical function in the ECMO group. These include differences between the groups in the use of steroids and neuromuscular blockers, presence of multiorgan dysfunction syndrome, sedation levels and muscle inactivity levels. The use of steroids post lung transplant was the same between the groups and therefore unlikely to be a contributory factor. Continuous neuromuscular blockade was not used in either group and is therefore unlikely to be a confounding factor and multiorgan dysfunction syndrome was not common in either group. Both groups had similar APACHE II scores at ICU admission, but the ECMO group required a longer period of sedation and mechanical ventilation and longer ICU LOS. A longer period of immobilization related to being on ECMO may have been a contributing factor to the lower functional level. Prolonged immobility is associated with decreased muscle protein synthesis and muscle atrophy, ${ }^{24}$ while critical illness is associated with an increased catabolic state with up-regulation of pro inflammatory mediators and changes in muscle composition leading to muscle weakness. ${ }^{25}$

The majority of subjects requiring ECMO underwent femoral cannulation, which may have been a barrier to early mobilization. Only two subjects mobilized out of bed while on ECMO, both of whom had dual lumen cannula. The majority were sedated and mechanically ventilated for the duration of ECMO, including all subjects that required ECMO post lung transplant. Previous studies have reported that femoral cannulation, sedation and mechanical ventilation are barriers to the early mobilization of ECMO patients, ${ }^{26,27}$ and there are no published studies to date describing the ambulation or out of bed rehabilitation of patients with femoral venoarterial ECMO. Over half of the subjects in our study underwent femoral venoarterial ECMO and required higher sedation levels than our protocol aims. Recent studies have reported higher levels of mobility while on ECMO in comparison to our study, but they included awake subjects that had upper body cannulation (dual lumen cannula) rather than femoral, venovenous rather than venoarterial ECMO, and not mechanically ventilated. ${ }^{28-30}$ Whereas our study included both pre and post lung transplant ECMO patients, these recent studies consisted mostly of bridge to lung transplant subjects. ${ }^{28-30}$

With physiotherapy rehabilitation, our ECMO subjects showed improvements in muscle strength and mobility status over time, with near normal muscle strength and independent walking by hospital discharge. These results compared favorably with those of 18 survivors of ARDS that underwent venovenous ECMO, ${ }^{31}$ in whom $83 \%$ described muscle weakness at hospital discharge and only $67 \%$ were ambulant. Our study cohort had similar APACHE II scores but a shorter ECMO duration [median $5 \mathrm{~d}$ (IQR 4-10) versus $11 \mathrm{~d}$ (IQR 4-16)]. The ARDS cohort may have experienced a longer period of immobilization as a result of the prolonged ECMO duration. Our study cohort also had a longer hospital LOS compared to the ARDS cohort (40.1 d vs $28.4 \mathrm{~d}$ ). Discharge from ICU and hospital 


\section{Physical Function Post ECMO}

is determined by medical readiness and is not reliant on the achievement of a specified functional status. If patients are medically ready for discharge but have not achieved premorbid level of function, they are transferred to another facility for in-patient rehabilitation. It may be that the lung transplant cohort had ongoing medical issues that were not present in the ARDS group and thus led to the increased hospital LOS. The longer hospital LOS in the lung transplant cohort may also have allowed for more rehabilitation once out of ICU, which may account for the higher level of physical function and higher discharge rate direct to home ( $86 \%$ vs $44 \%)$.

HRQOL in our ECMO survivors was impaired at hospital discharge compared to that of Australian norms, revealing problems with work or other daily activities because of physical health and pain. HRQOL was only measured at hospital discharge in our ECMO cohort, which may partly account for the lower scores. Other studies reporting on HRQOL in ECMO survivors ${ }^{32}$ have reported significant improvements in HRQOL as time from hospital discharge increases, and longer-term follow-up is warranted in this population. HRQOL was not assessed in the non-ECMO lung transplant subjects, therefore comparison between the groups is not possible but is warranted in future studies.

Among the 14 survivors, 7 (50\%) reported complications involving the lower extremity. Six subjects (43\%) developed vascular complications, which occurred in over half of subjects (67\%, 6 of 9 subjects) who had femoral venoarterial ECMO. This rate is higher than that reported in a study of 101 subjects requiring femoral venoarterial ECMO. ${ }^{33}$ Aziz et $\mathrm{al}^{33}$ reported a vascular complication rate of $18 \%$, using cannulation techniques very similar to that described in our study. Our cohort was entirely composed of lung transplant patients, whereas the etiology of the subjects in the Aziz et al ${ }^{33}$ study is unclear but appears to be a mix of cardiogenic shock and patients with ARDS. Impaired wound healing after lung transplant secondary to immunosuppression and the need for femoral artery surgical repair after ECMO arterial decannulation may be related to the higher complication rate seen in our lung transplant cohort.

Although our study showed no significant difference in 6MWD or HRQOL between subjects with a leg complication and those without, this may be due to the small sample size and warrants further investigation in a larger cohort of ECMO patients. The impact of leg complications on participation in post-transplant rehabilitation also was minimal, with only minor modifications to the exercise program required. A small percentage (10\%) of readmissions in the year following lung transplantation was directly related to vascular complications related to ECMO. Longer-term monitoring of these leg complications is required to determine whether these complications persisted or lessened over time. In addition, the impact of early, more intensive rehabilitation during the ICU stay on physical function, HRQOL, and leg complications warrants further investigation.

Our study has several limitations. First, our study is a single-center retrospective study. Second, our population of subjects was a mixed cohort receiving both venoarterial and venovenous femoral and dual-lumen cannulation for a variety of medical and post-lung transplant causes. This was, however, representative of the population of patients requiring lung transplantation. Detailed evaluation of the different populations is warranted in larger trials. Although our study is the first to report on early physical function and lower limb complications in subjects before and after lung transplantation, the numbers are too small to draw definitive conclusions and preclude any detailed subgroup analyses. The multiple regression analysis is also limited by the small sample size, and results should be viewed as hypothesis-generating rather than generalizable to the wider ECMO population. HRQOL and strength were only assessed in the ECMO group and were not available for the non-ECMO subjects, so comparison could not be made between the groups for these measures. Finally, level of function prior to the implementation of ECMO was not objectively assessed as subjects often presented acutely or were transferred from other hospitals. However, a retrospective review of the medical history revealed that only 1 subject was bed-bound in the $48 \mathrm{~h}$ prior to ECMO initiation. Potential benefits from this study include new knowledge about the early physical function, leg complications, and HRQOL of subjects who have undergone ECMO before and after lung transplantation. Furthermore, this study identifies increased hospital LOS and worse physical function in lung transplant recipients requiring ECMO versus those who did not require ECMO. This may assist in the development of targeted treatment guidelines for this patient population, which currently do not exist.

\section{Conclusion}

This was the first study of early physical function after ECMO as a rescue therapy for subjects before or after lung transplantation, with comparison to non-ECMO lung transplant recipients. In this study population, subjects requiring ECMO had poor physical function at ICU discharge, but this improved by the time of hospital discharge, with the majority of subjects discharged directly to home. A longer period of mechanical ventilation and longer ICU and hospital LOS, along with lower physical function at ICU and hospital discharge, were observed in the ECMO group compared to the non-ECMO group; this may be related to the ECMO cannulation strategy and the level of sedation in the ECMO group. ICU LOS was the only 


\section{Physical Function Post ECMO}

significant predictor of physical function at hospital discharge. HRQOL was poor at hospital discharge in the ECMO subjects and warrants further investigation with longer-term follow-up, as does the incidence and impact of complications involving the lower limb in subjects receiving femoral ECMO.

\section{REFERENCES}

1. Yusen RD, Edwards LB, Kucheryavaya AY, Benden C, Dipchand AI, Goldfarb SB, et al. The registry of the International Society for Heart and Lung Transplantation: thirty-second official adult lung and heart-lung transplantation report-2015; focus theme: early graft failure. J Heart Lung Transplant 2015;34(10):1264-1277.

2. Lindstrom SJ, Pellegrino VA, Butt WW. Extracorporeal membrane oxygenation. Med J Aust 2009;191(3):178-182.

3. Chiumello D, Coppola S, Froio S, Colombo A, Del Sorbo L. Extracorporeal life support as bridge to lung transplantation: a systematic review. Crit Care 2015;19:19.

4. Gulak BC, Hirji SA, Hartwig MG. Bridge to lung transplantation and rescue post-tranplant: the expanding role of extracorporeal membrane oxygenation. J Thorac Dis 2014;6(8):1070-1079.

5. Javidfar J, Brodie D, Iribarne A, Jurado J, Lavelle M, Brenner K, et al. Extracorporeal membrane oxygenation as a bridge to lung transplantation and recovery. J Thorac Cardiovasc Surg 2012;144(3):716-721.

6. Toyoda Y, Bhama JK, Shigemura N, Zaldonis D, Pilewski J, Crespo $\mathrm{M}$, et al. Efficacy of extracorporeal membrane oxygenation as a bridge to lung transplantation. J Thorac Cardiovasc Surg 2013;145(4): 1065-1070.

7. Bittner HB, Lehmann S, Rastan A, Garbade J, Binner C, Mohr FW, et al. Outcome of extracorporeal membrane oxygenation as a bridge to lung transplantation and graft recovery. Ann Thorac Surg 2012; 94(3):942-949.

8. Wigfield CH, Lindsey JD, Steffens TG, Edwards NM, Love RB. Early institution of extracorporeal membrane oxygenation for primary graft dysfunction after lung transplantation improves outcome. J Heart Lung Transplant 2007;26(4):331-338.

9. Hayes K, Holland AE, Pellegrino VA, Leet AS, Fuller LM, Hodgson CL. Physical function after extracorporeal membrane oxygenation in patients pre or post heart transplantation - An observational study. Heart Lung 2016;45(6):525-531.

10. Aubron C, Cheng AC, Pilcher D, Leong T, Magrin G, Cooper DJ, et al. Factors associated with outcomes of patients on extracorporeal membrane oxygenation support: a 5-year cohort study. Crit Care 2013;17(2):R73.

11. Javidfar J, Brodie D, Wang D, Ibrahimiye AN, Yang J, Zwischenberger JB, et al. Use of bicaval dual-lumen catheter for adult venovenous extracorporeal membrane oxygenation. Ann Thorac Surg 2011;91(6):1763-1768.

12. Sessler CN, Gosnell MS, Grap MJ, Brophy GM, O'Neal PV, Keane KA, et al. The Richmond Agitation-Sedation Scale: validity and reliability in adult intensive care unit patients. Am J Respir Crit Care Med 2002;166(10):1338-1344.

13. Ramirez M. Multiple organ dysfunction syndrome. Curr Probl Pediatr Adolesc Health Care 2013;43(10):273-277.

14. Hodgson C, Needham D, Haines K, Bailey M, Ward A, Harrold M, et al. Feasibility and inter-rater reliability of the ICU Mobility Scale. Heart Lung 2014;43(1):19-24.

15. Holland AE, Spruit MA, Troosters T, Puhan MA, Pepin V, Saey D, et al. An official European Respiratory Society/American Thoracic Society technical standard: field walking tests in chronic respiratory disease. Eur Respir J 2014;44(6):1428-1446.
16. Kleyweg RP, van der Meche FG, Schmitz PI. Interobserver agreement in the assessment of muscle strength and functional abilities in Guillain-Barre syndrome. Muscle Nerve 1991;14(11):1103-1109.

17. Ali NA, O'Brien JM, Jr., Hoffmann SP, Phillips G, Garland A, Finley JC, et al. Acquired weakness, handgrip strength, and mortality in critically ill patients. Am J Respir Crit Care Med 2008;178(3): 261-268.

18. De Jonghe B, Sharshar T, Lefaucheur JP, Authier FJ, Durand-Zaleski I, Boussarsar M, et al. Paresis acquired in the intensive care unit: a prospective multicenter study. JAMA 2002;288(22):28592867.

19. Ries AL, Bauldoff GS, Carlin BW, Casaburi R, Emery CF, Mahler DA, et al. Pulmonary rehabilitation: Joint ACCP/AACVPR evidencebased clinical practice guidelines. Chest 2007;131(5 Suppl):4S-42S.

20. Hawthorne G, Osborne RH, Taylor A, Sansoni J. The SF36 version 2: critical analyses of population weights, scoring algorithms and population norms. Qual Life Res 2007;16(4):661-673.

21. Hawthorne G. Measuring incontinence in Australia. Melbourne: Commonwealth of Australia;2006:49.

22. Bernard S, LeBlanc P, Whittom F, Carrier G, Jobin J, Belleau R, et al. Peripheral muscle weakness in patients with chronic obstructive pulmonary disease. Am J Respir Crit Care Med 1998;158(2): 629-634.

23. Troosters T, Langer D, Vrijsen B, Segers J, Wouters K, Janssens W, et al. Skeletal muscle weakness, exercise tolerance and physical activity in adults with cystic fibrosis. Eur Respir J 2009;33(1):99-106.

24. Paddon-Jones D, Sheffield-Moore M, Cree MG, Hewlings SJ, Aarsland A, Wolfe RR, et al. Atrophy and impaired muscle protein synthesis during prolonged inactivity and stress. J Clin Endocrinol Metab 2006;91(12):4836-4841.

25. Puthucheary ZA, Rawal J, McPhail M, Connolly B, Ratnayake G, Chan P, et al. Acute skeletal muscle wasting in critical illness. JAMA 2013;310(15):1591-1600.

26. Abrams D, Javidfar J, Farrand E, Mongero LB, Agerstrand CL, Ryan $\mathrm{P}$, et al. Early mobilization of patients receiving extracorporeal membrane oxygenation: a retrospective cohort study. Crit Care 2014; 18(1):R38.

27. Ko Y, Cho YH, Park YH, Lee H, Suh GY, Yang JH, et al. Feasibility and safety of early physical therapy and active mobilization for patients on extracorporeal membrane oxygenation. ASAIO J 2015; 61(5):564-568.

28. Garcia JP, Kon ZN, Evans C, Wu Z, Iacono AT, McCormick B, et al. Ambulatory veno-venous extracorporeal membrane oxygenation: innovation and pitfalls. J Thorac Cardiovasc Surg 2011;142(4):755-761.

29. Rehder KJ, Turner DA, Hartwig MG, Williford WL, Bonadonna D, Walczak RJ, Jr., et al. Active rehabilitation during extracorporeal membrane oxygenation as a bridge to lung transplantation. Respir Care 2013;58(8):1291-1298.

30. Turner DA, Cheifetz IM, Rehder KJ, Williford WL, Bonadonna D, Banuelos SJ, et al. Active rehabilitation and physical therapy during extracorporeal membrane oxygenation while awaiting lung transplantation: a practical approach. Crit Care Med 2011;39(12):2593-2598.

31. Hodgson CL, Hayes K, Everard T, Nichol A, Davies AR, Bailey MJ, et al. Long-term quality of life in patients with acute respiratory distress syndrome requiring extracorporeal membrane oxygenation for refractory hypoxaemia. Crit Care 2012;16(5):R202.

32. Combes A, Leprince P, Luyt CE, Bonnet N, Trouillet JL, Leger P, et al. Outcomes and long-term quality-of-life of patients supported by extracorporeal membrane oxygenation for refractory cardiogenic shock. Crit Care Med 2008;36(5):1404-1411.

33. Aziz F, Brehm CE, El-Banyosy A, Han DC, Atnip RG, Reed AB. Arterial complications in patients undergoing extracorporeal membrane oxygenation via femoral cannulation. Ann Vasc Surg 2014; 28(1):178-183 